\title{
Phantom field fluctuation induced Higgs effect
}

\author{
A. Patkós ${ }^{a, b}$ \\ ${ }^{a}$ Department of Atomic Physics, Eötvös University, H-1117 Budapest, Hungary \\ ${ }^{\mathrm{b}}$ Research Group for Statistical Physics of the Hungarian Academy of Sciences, \\ H-1117 Budapest, Hungary \\ Zs. Szép \\ Research Institute for Solid State Physics and Optics of the Hungarian Academy \\ of Sciences, H-1525 Budapest, Hungary
}

\begin{abstract}
Symmetry breaking solutions are investigated in the $N \rightarrow \infty$ limit for the ground state of a system consisting of a Lorentz-scalar, $N$ component "phantom" field and an $O(N)$ singlet. The most general form of $O(N) \times Z_{2}$ invariant quartic interaction is considered. The non-perturbatively renormalised solution demonstrates the possibility for $Z_{2}$ symmetry breaking induced by phantom fluctuations. It becomes also evident that the strength of the "internal" dynamics of the N-component field tunes away the ratio of the Higgs condensate and the Higgs mass from its perturbative (nearly tree-level) expression.
\end{abstract}

Key words: Higgs mass, phantom field, Dyson-Schwinger equations, large N approximation

PACS: 11.10.Wx, 11.10.Gh, 12.38.Cy

\section{Introduction}

Scalar fields are proposed at present with renewed intensity as minimal, phenomenologically motivated complements to the Standard Model. The simplest mechanism for electroweak symmetry breaking is realised by introducing a renormalisable biquadratic

Email addresses: patkos@ludens.elte.hu (A. Patkós), szepzs@achilles.elte.hu (Zs. Szép). 
coupling of the Higgs field to a "hidden scalar" (the phantom field) which acquires nonzero vacuum expectation value $[1,2]$. Postulation of two extra scalars with appropriate dynamics might provide the minimal framework for the particle physics interpretation of the cosmologically well-established facts of missing luminous mass and of inflationary density fluctuations [3,4]. A scalar inflaton coupled to sterile right-handed neutrinos offers another cosmologically motivated minimal extension of the standard model, which accounts also for neutrino oscillation phenomena and the fermion-asymmetry of the universe [5].

A general background to this evolution is provided by low energy field theories constructed on string vacua showing maximal compatibility with the particle content of the Standard Model. Appearance of extra scalars is characteristic for all these constructions. A consequence of possible physical significance is that the natural cut-off scale to be applied for the top quark contribution to the Higgs mass would be raised considerably [6]. Consequences of the existence of a phantom sector on the precision electroweak tests of the Standard Model are being systematically explored [7]. The history of such investigations goes back to the 80's [8].

The goal of this letter is to illustrate the substantial influence of an extended scalar sector on the symmetry breaking vacuum features through the results of an exact, nonperturbatively renormalised large $N$ analysis. We propose the following embedding of the Higgs sector of the Standard Model into a wider set of elementary scalar fields. The gauged self-interacting scalar $S U(2)$ doublet is coupled also to a set of scalar fields which form an $N$-component vector under a hidden internal $O(N)$ symmetry. The coupling preserves the $S U(2)$ symmetry of the standard gauge model. After fixing 3 components of the complex Higgs doublet to zero a $Z_{2}$ invariance of the Higgs field is left. The phantom $O(N)$ vector field has some extra $O(N)$ invariant non-linear interaction on its own. With appropriately chosen $N$-scaling of the quartic couplings the renormalised $N=\infty$ solutions will be presented. We shall demonstrate that the non-perturbative renormalisation of the solution leads to a non-trivial $Z(2)$ symmetry breaking Higgs condensate in a large range of the renormalised couplings of the model even if no negative renormalised squared mass is introduced. It will be shown that also essential deviations might appear in the relation of the Higgs mass to the strength of the Higgs condensate, relative to the leading order large $N$ estimate derived in the $O(N)$ symmetric linear sigma model.

The present model is a generalisation of the $O(N+M)$ symmetric model studied by Chivukula et al. in 1991-1993 [9]. Another extension of the Higgs sector was investigated in [10], where consequences of the mirror world assumption were studied. Here the general model will be solved by taking the large $N$ limit of the renormalised Dyson-Schwinger equations for the exact $n$-point functions. The renormalisation process will follow an approach advocated recently in the context of 2PI approximate solution of scalar field theories [11], but we emphasise that it represents a rather non-trivial implementation of the strategy of nonperturbative renormalisation. 


\section{Leading order formal solution}

The Lagrangian to be solved at $N=\infty$ describes the most general renormalisable $S U(2) \times$ $O(N)$ symmetric interaction of an $N$-component (and $\mathrm{SU}(2)$ singlet) field $\psi_{i}, i=1, . ., N$ with a gauged $S U(2)$ doublet (and $O(N)$ singlet) $\Phi$ :

$$
\begin{aligned}
L\left[\psi_{i}, \phi\right]= & \frac{1}{2}\left(D_{\mu} \Phi\right)^{\dagger} D^{\mu} \Phi+\frac{1}{2}\left(\partial_{\mu} \psi_{i}\right)^{2}-\frac{1}{2} m_{2}^{2} \psi_{i}^{2}-\frac{1}{2} m_{3}^{2} \Phi^{\dagger} \Phi \\
& -\frac{\lambda_{1}}{24 N}\left(\psi_{i}^{2}\right)^{2}-\frac{\lambda_{2}}{24 N}\left(\Phi^{\dagger} \Phi\right)^{2}-\frac{\lambda_{3}}{12 N} \psi_{i}^{2} \Phi^{\dagger} \Phi .
\end{aligned}
$$

The present parametrisation is a generalisation of that used in [9], where only a unique quartic coupling $\sim\left(\psi_{i}^{2}+\Phi^{\dagger} \Phi\right)^{2}$ was considered.

We shall investigate the usual symmetry breaking pattern, where the lower real part of the complex $S U(2)$ doublet gets a nonzero classical value $\sqrt{N} v$, and the other three components are gauge transformed away (unitary gauge). The shift in the Higgs field then breaks the $Z_{2}$ symmetry related to the remaining reflection symmetry of the Higgs field. When gauge fields are set to zero the resulting shifted Lagrangian density is the following:

$$
\begin{aligned}
L_{\text {free }}\left[\psi_{i}, \sigma\right]= & \frac{1}{2}\left(\partial_{\mu} \sigma\right)^{2}+\frac{1}{2}\left(\partial_{\mu} \psi_{i}\right)^{2}-\frac{1}{2} m_{2}^{2} \psi_{i}^{2}-\frac{1}{2} m_{3}^{2}\left(\sigma^{2}+2 \sigma \sqrt{N} v+N v^{2}\right), \\
L_{\text {int }}\left[\psi_{i}, \sigma\right]= & -\frac{\lambda_{1}}{24 N}\left(\psi_{i}^{2}\right)^{2}-\frac{\lambda_{2}}{24 N}\left(\sigma^{2}+2 \sigma \sqrt{N} v+N v^{2}\right)^{2} \\
& -\frac{\lambda_{3}}{12 N} \psi_{i}^{2}\left(\sigma^{2}+2 \sigma \sqrt{N} v+N v^{2}\right) .
\end{aligned}
$$

The large $N$ solutions will be constructed with help of the Dyson-Schwinger (DS) equations which are generated by the master equation

$$
\frac{\delta \Gamma}{\delta \varphi_{U}}=\frac{\delta S_{c l}}{\delta \varphi_{U}}\left[\varphi_{A}+G_{A B} \frac{\delta}{\delta \varphi_{B}}\right]
$$

and its functional derivatives [12]. The right hand side is evaluated by replacing in the expression of the functional derivative of the classical action the field $\varphi_{U}$ by $\varphi_{U}+G_{U V} \delta / \delta \varphi_{V}$ and the whole expression is applied to the unity in the field space. $\Gamma$ is the effective action, $\varphi_{U}$ is a generic field variable, $G_{A B}$ is the two-point function of the generic fields $\varphi_{A}$ and $\varphi_{B}$ (deWitt's convention is used for the index $A, B, \ldots$ ). Note, that one should include into the intermediate steps of the derivation all possible $(A, B)$ pairs of indices for $G_{A B}$, the corresponding external currents are set to zero only at the very end.

Below we give the unrenormalised equations which determine the vacuum condensate $v$, the propagators $G_{\sigma \sigma}(x, y)=: G_{\sigma}(x, y), G_{\psi_{m} \psi_{n}}(x, y)=: \delta_{n m} G_{\psi}(x, y)$ and the relevant 
3-point function $\Gamma_{\psi_{n} \psi_{m} \sigma}(x, y, z)=\delta_{n m} \Gamma_{\psi \psi \sigma}(x, y, z)$. The LO $(N=\infty)$ equation for the vacuum expectation value $v$ looks rather simple:

$$
-\left.\frac{\delta \Gamma}{\delta \sigma}\right|_{\mid \varphi_{U}=0}=\sqrt{N} v\left[m_{3}^{2}+\frac{\lambda_{2}}{6} v^{2}+\frac{\lambda_{3}}{6} T_{\psi}\right]=0, \quad T_{\psi}=\int_{k} G_{\psi}(k) .
$$

The LO $\psi$ propagator is obtained as $\delta^{2} \Gamma / \delta \psi \delta \psi=: i G_{\psi}^{-1}(p)$ in the following form

$$
i G_{\psi}^{-1}(p)=p^{2}-m_{2}^{2}-\frac{\lambda_{3}}{6} v^{2}-\frac{\lambda_{1}}{6} T_{\psi}
$$

Since the tadpole is momentum independent the propagator can be parametrised as $G_{\psi}(p)=i /\left(p^{2}-m_{\psi}^{2}\right)$ where $m_{\psi}^{2}$ is determined by the gap-equation

$$
m_{\psi}^{2}=m_{2}^{2}+\frac{\lambda_{3}}{6} v^{2}+\frac{\lambda_{1}}{6} T_{\psi}
$$

Finally, the closed system determining the propagator $G_{\sigma}$ and the 3-point function $\Gamma_{\psi \psi \sigma}$ is derived by taking the corresponding second and third functional derivatives of the effective action:

$$
\begin{aligned}
i G_{\sigma}^{-1}(p)= & p^{2}-m_{3}^{2}-\frac{\lambda_{2}}{2} v^{2}-\frac{\lambda_{3}}{6} T_{\psi} \\
& -i \sqrt{N} v \frac{\lambda_{3}}{6} \int_{k} G_{\psi}(p-k) G_{\psi}(k) \Gamma_{\psi \psi \sigma}(p-k, k,-p), \\
\Gamma_{\psi \psi \sigma}(p, q,-p-q)= & -\frac{\lambda_{3} v}{3 \sqrt{N}} \\
& -i \frac{\lambda_{1}}{6} \int_{k} G_{\psi}(p+q-k) G_{\psi}(k) \Gamma_{\psi \psi \sigma}(p+q-k, k,-p-q) .
\end{aligned}
$$

We note that $\Gamma_{\psi \psi \sigma}$ was calculated from the master equation of $\delta \Gamma / \delta \psi$. In the last equation the expression of the right hand side shows that $\Gamma_{\psi \psi \sigma}(p, q,-p-q)$ on the left depends only on the momentum of $\sigma$. From this it promptly follows for the 3-point function that

$$
\Gamma_{\psi \psi \sigma}(p, q,-p-q)=-\frac{\lambda_{3} v}{3 \sqrt{N}} \frac{1}{1-\frac{\lambda_{1}}{6} I_{\psi}(p+q)}, \quad I_{\psi}(p)=-i \int_{k} G_{\psi}(p-k) G_{\psi}(k)
$$

which leads (by exploiting Eq.(4)) to

$$
i G_{\sigma}^{-1}(p)=p^{2}-\frac{\lambda_{2}}{3} v^{2}-\frac{\lambda_{3}^{2}}{18} v^{2} \frac{I_{\psi}(p)}{1-\frac{\lambda_{1}}{6} I_{\psi}(p)} .
$$

The renormalisation algorithm of the equations (4), (5) and (7) is rather non-trivial. The result can be stated, however, very simply: i) one should replace in these equations the bare couplings $m_{i}^{2}, \lambda_{i}$ by the corresponding renormalised ones; ii) the quadratically divergent tadpole integral $T_{\psi}$ and the logarithmically divergent $I_{\psi}$ should be replaced by the finite 
parts $T_{\psi, F}, I_{\psi, F}$ arising after subtracting the divergent pieces. It is worth noting, that by the renormalisation of $\lambda_{i}$ the 3-point function $\Gamma_{\psi \psi \sigma}$ is actually a finite asymptotically decreasing function of the $\sigma$-momentum. As a consequence no divergence proportional to $p^{2}$ appears in $G_{\sigma}^{-1}$, therefore there is no need for infinite field renormalisation.

The derivation of the non-trivial relation of the renormalised couplings to the unrenormalised ones will be outlined in the next section. We apply cut-off regularisation to the divergent integrals $T_{\psi}(M)$ and $I_{\psi}(p, M)$, which are evaluated after Euclidean continuation of the variables:

$$
\begin{aligned}
T_{\psi}(M) & \equiv \int_{k} \frac{i}{k^{2}-M^{2}}=\frac{\Lambda^{2}}{16 \pi^{2}}-\frac{M^{2}}{16 \pi^{2}} \ln \frac{e \Lambda^{2}}{M_{0}^{2}}+T_{\psi, F}(M), \\
I_{\psi}(p, M) & \equiv \int_{k} \frac{i}{\left(k^{2}-M^{2}\right)\left((p-k)^{2}-M^{2}\right)}=-\frac{1}{16 \pi^{2}} \ln \frac{e \Lambda^{2}}{M_{0}^{2}}+I_{\psi, F}(p, M),
\end{aligned}
$$

where the scale $M_{0}$ is introduced to eliminate the $M$-dependence of the cut-off dependent terms. The renormalisation will be achieved by compensating the cut-off dependence explicitly written out above by appropriately chosen coupling counterterms.

\section{Renormalisation}

The ultraviolet renormalisation of the equations (4), (5) and (7) requires a fresh analysis of the counter-term resummation, since the unrenormalised equations do not suggest any transparent formula similar to the simpler case of the $O(N)$ model (see e.g. [13]).

Below we shall apply the iterative renormalisation of [11] to our case. The renormalisation of the equation of state and pion propagator (gap-equation) is the usual superdaisy renormalisation described in details in [11]. The renormalisation of the system (7) is a little bit more involved. First one rewrites the previous equations by making explicit the counterterms. Since the derivation of the DS equations starts from the classical action, there are explicit Lagrangian parameters present which we have to split as $m_{i}^{2}=m_{i, R}^{2}+\delta m_{i}^{2}, \lambda_{i}=\lambda_{i, R}+\delta \lambda_{i}$. But the effect of the counterterm does not reduce merely to this. Their consistent application implies that the 3-point function entering the DS equation of the sigma propagator is the renormalised one. This statement can be easily checked when using in the equation of the sigma propagator the iterative solution of the equation of the renormalised 3-point function:

$$
\begin{aligned}
\Gamma_{\psi \psi \sigma}(p, q,-p-q)= & -\frac{\lambda_{3, R}+\delta \lambda_{3}}{3 \sqrt{N}} v \\
& -i \frac{\lambda_{1, R}+\delta \lambda_{1}}{6} \int_{k} G_{\psi}(p+q-k) G_{\psi}(k) \Gamma_{\psi \psi \sigma}(p+q-k, k,-p-q) .
\end{aligned}
$$


At a given order of the iteration we recover the chain of bubble diagrams including the counterterm diagrams of the usual perturbation theory at the corresponding order of the loop expansion.

Next we subtract Eq. (4) from the first equation of (7) to obtain for the renormalised $i G_{\sigma}^{-1}$ the expression

$$
i G_{\sigma}^{-1}(p)=p^{2}-\frac{\lambda_{2, R}+\delta \lambda_{2}}{3} v^{2}-i \sqrt{N} v \frac{\lambda_{3, R}+\delta \lambda_{3}}{6} \int_{k} G_{\psi}(p-k) G_{\psi}(k) \Gamma_{\psi \psi \sigma}(p-k, k,-p) .
$$

The iterative renormalisation consists of assuming infinite series expansion for the counterterms and determining them by requiring the cancellation of the divergencies generated at each iteration step of the solution of the coupled equations of $G_{\sigma}$ and $\Gamma_{\psi \psi \sigma}$.

In the first iteration of the vertex function one substitutes into the propagator equation the tree-level value of the 3-point vertex which yields the requirement:

$$
\frac{\delta \lambda_{2}^{(1)}}{3} v^{2}+\frac{\lambda_{3, R}^{2}}{18} v^{2}\left(-i \int_{k} G_{\psi}(p-k) G_{\psi}(k)\right)_{\text {div }}=0 .
$$

The same sort of first iteration is done also in the integral equation of $\Gamma_{\psi \psi \sigma}$ and the two together lead to

$$
\delta \lambda_{2}^{(1)}=-\frac{\lambda_{3, R}^{2}}{6} I_{\psi, d i v}, \quad \delta \lambda_{3}^{(1)}=-\frac{\lambda_{1, R} \lambda_{3, R}}{6} I_{\psi, d i v}
$$

With this step also the finite parts are determined, which are substituted into the second iteration. This step produces on the right hand side of the expression $i G_{\psi}^{-1}$ the following divergent piece in the full expression, which has to vanish:

$$
\left[\frac{\delta \lambda_{2}^{(2)}}{3}+2 \frac{\lambda_{3, R} \delta \lambda_{3}^{(1)}}{18} I_{\psi, d i v}+\frac{\lambda_{3, R}}{9} I_{\psi, F}(p)\left(\delta \lambda_{3}^{(1)}+\frac{\lambda_{1, R} \lambda_{3, R}}{6} I_{\psi, d i v}\right)+\frac{\lambda_{1, R} \lambda_{3, R}^{2}}{108} I_{\psi, \text { div }}^{2}\right] v^{2}=0
$$

It is important that the subdivergence proportional to the finite (possibly temperature or density dependent) $I_{\psi, F}$ automatically vanishes in view of the previous iteration step. This feature of subdivergence cancellation is generally valid for each iteration step. The remaining divergent terms determine the second term in the infinite series of $\delta \lambda_{2}$ :

$$
\delta \lambda_{2}^{(2)}=\frac{\lambda_{3, R}^{2} \lambda_{1, R}}{36} I_{\psi, d i v}^{2}
$$

The second iteration of the equation of $\Gamma_{\psi \psi \sigma}$ determines $\delta \lambda_{3}^{(2)}$ and $\delta \lambda_{1}^{(1)}=-\lambda_{1, R}^{2} I_{\psi, \text { div }} / 6$. With simple induction the following general recursions are found for the subsequent iterative terms of the counterterm series:

$$
\delta \lambda_{1}^{(n)}=-\delta \lambda_{1}^{(n-1)} \frac{\lambda_{1, R}}{6} I_{\psi, d i v}, \quad \delta \lambda_{2}^{(n)}=-\delta \lambda_{3}^{(n-1)} \frac{\lambda_{3, R}}{6} I_{\psi, \text { div }}, \quad \delta \lambda_{3}^{(n)}=-\delta \lambda_{1}^{(n-1)} \frac{\lambda_{3, R}}{6} I_{\psi, \text { div }}
$$


where $n \geq 1$ and $\delta \lambda_{1}^{(0)}:=\lambda_{1, R}, \delta \lambda_{3}^{(0)}:=\lambda_{3, R}$.

These equations imply the following non-perturbative renormalisation formulae:

$$
\frac{1}{\lambda_{1}}=\frac{1}{\lambda_{1, R}}+\frac{1}{6} I_{\psi, d i v}, \quad \frac{\lambda_{3}}{\lambda_{1}}=\frac{\lambda_{3, R}}{\lambda_{1, R}}, \quad \lambda_{2}-\frac{\lambda_{3}^{2}}{\lambda_{1}}=\lambda_{2, R}-\frac{\lambda_{3, R}^{2}}{\lambda_{1, R}} .
$$

In addition to these relations between bare and renormalised couplings we also obtain the renormalised sigma self-energy which is nothing but the expansion of the last term in (9) in powers of $\lambda_{1}$ up to the given order of the iteration (of course, the couplings and the bubble integral have to be replaced by their renormalised expressions).

It is easy to check that provided we would have known a priori these relations, they indeed ensure the simple form of the renormalised expression of (9) announced at the end of the previous section. The mass of the Higgs particle is determined by the pole of equation (9) $\left(i G^{-1}\left(p^{2}=M_{\sigma}^{2}\right)=0\right)$

$$
M_{\sigma}^{2}=\frac{v^{2}}{3}\left[\left(\lambda_{2, R}-\frac{\lambda_{3, R}^{2}}{\lambda_{1, R}}\right)+\frac{\lambda_{3, R}^{2}}{\lambda_{1, R}^{2}} \frac{1}{\lambda_{1, R}^{-1}-I_{\psi, F}\left(p^{2}=M_{\sigma}^{2}\right) / 6}\right] .
$$

The renormalised quartic couplings are used also in the iterative renormalisation of the equations for the order parameter $v$ and of $G_{\psi}$ in which the tadpole is calculated with a self-consistent propagator. The mass counterterms $\delta m_{2}^{2}$ and $\delta m_{3}^{2}$ are determined as:

$$
\begin{gathered}
\delta m_{2}^{2(n)}=-\frac{\delta \lambda_{1}^{(n-1)}}{6} \frac{\Lambda^{2}}{16 \pi^{2}}+m_{2, R}^{2} \frac{\delta \lambda_{3}^{(n)}}{\lambda_{3, R}}=-\frac{\delta \lambda_{1}^{(n-1)}}{6} \frac{\Lambda^{2}}{16 \pi^{2}}+m_{2, R}^{2} \frac{\delta \lambda_{1}^{(n)}}{\lambda_{1, R}} \\
\delta m_{3}^{2(n)}=-\frac{\delta \lambda_{3}^{(n-1)}}{6} \frac{\Lambda^{2}}{16 \pi^{2}}+m_{2, R}^{2} \frac{\delta \lambda_{2}^{(n)}}{\lambda_{3, R}}=-\frac{\delta \lambda_{3}^{(n-1)}}{6} \frac{\Lambda^{2}}{16 \pi^{2}}+m_{2, R}^{2} \frac{\delta \lambda_{1}^{(n)} \lambda_{3, R}}{\lambda_{1, R}^{2}}
\end{gathered}
$$

implying

$$
\frac{m_{2}^{2}}{\lambda_{1}}+\frac{\Lambda^{2}}{96 \pi^{2}}=\frac{m_{2, R}^{2}}{\lambda_{1, R}}, \quad m_{3}^{2}-m_{2}^{2} \frac{\lambda_{3}}{\lambda_{1}}=m_{3, R}^{2}-m_{2, R}^{2} \frac{\lambda_{3, R}}{\lambda_{1, R}} .
$$

The second equalities in both equations of (20) follow when one exploits (17). Making use of these renormalised relations in (4) and (5) one easily derives for the renormalised expression of the vacuum expectation value the following formula:

$$
\frac{1}{6} v^{2}=\left[-m_{3, R}^{2}+\left(m_{2, R}^{2}-m_{\psi}^{2}\right) \frac{\lambda_{3, R}}{\lambda_{1, R}}\right]\left(\lambda_{2, R}-\frac{\lambda_{3, R}^{2}}{\lambda_{1, R}}\right)^{-1} .
$$

\section{Discussion}

The expressions for the Higgs mass (19) and the Higgs condensate (22) show that the influence of the phantom sector might be quite strong on both. In order to make the 
discussion more transparent below we shall choose

$$
\lambda_{2, R}=\lambda_{3, R} \equiv \lambda, \quad \lambda_{1, R} \equiv \lambda+\lambda^{\prime}
$$

In this case the gap equation (6) which determines $m_{\psi}$ reads after the elimination of $v^{2}$ as

$$
m_{\psi}^{2}=m_{2, R}^{2}-m_{3, R}^{2}+\frac{\lambda^{\prime}}{6} T_{\psi, F}, \quad T_{\psi, F}=\frac{m_{\psi}^{2}}{16 \pi^{2}} \ln \left(\frac{e m_{\psi}^{2}}{M_{0}^{2}}\right) .
$$

which has the approximate solution (for small $\lambda^{\prime}$ )

$$
m_{\psi}^{2} \approx \frac{m_{2, R}^{2}-m_{3, R}^{2}}{1-\frac{\lambda^{\prime}}{96 \pi^{2}} \ln \frac{e\left(m_{2, R}^{2}-m_{3, R}^{2}\right)}{M_{0}^{2}}}
$$

For sufficiently large renormalisation scale $M_{0}$ one has $0<m_{\psi}^{2}<m_{2, R}^{2}-m_{3, R}^{2}$. The Higgs condensate has the simple equation

$$
\frac{\lambda}{6} v^{2}=-m_{3, R}^{2}+\frac{\lambda}{\lambda^{\prime}}\left(m_{2, R}^{2}-m_{3, R}^{2}-m_{\psi}^{2}\right)
$$

Depending on the ratio of the two quartic couplings and the differences in the renormalised mass parameters we see that the phantom fluctuations might produce a stable $Z_{2}$ symmetry breaking condensate. This mechanism is similar to the dynamical symmetry breaking of Coleman and Weinberg [14]. The role of the electromagnetic field in their example is played here by $\psi_{i}, \lambda^{\prime}$ and the dynamically determined $m_{\psi}^{2}$. Also the Higgs mass

$$
M_{\sigma}^{2}=\frac{v^{2}}{3} \frac{\lambda}{\lambda+\lambda^{\prime}}\left[\lambda^{\prime}+\frac{\lambda}{1-\frac{\lambda+\lambda^{\prime}}{6} I_{\psi, F}\left(p^{2}=M_{\sigma}^{2}\right)}\right]
$$

depends in a substantial way on both couplings which would give more flexibility in Higgs phenomenology.

In conclusion, we have presented an $O(N)$ symmetric model of the phantom world interacting with the SM Higgs field, which was solved in the $N=\infty$ limit. The solution obtained with iterative renormalisation represents a non-trivial generalisation of the wellknown example of the pure $O(N)$ model. The method can be applied to a large variety of models containing several multiplets with large number of components.

The existence of symmetry breaking solutions stabilised by the phantom sector fluctuations was pointed out. In this respect we emphasise that Eqs. (24), (26) and (27) are valid also at finite temperature, if $I_{\psi, F}$ and $T_{\psi, F}$ are evaluated accordingly. They can serve for investigating the nature of finite temperature symmetry restoration in the generalised Higgs sector.

Non-perturbative (lattice) studies at finite $N$ could clarify if our findings are generically valid also for finite number of phantom field components. 


\section{Acknowledgements}

We thank the participants of a Croatian-Hungarian Bilateral Workshop in Theoretical Physics for valuable discussions on the first version of this material. Work supported by Hungarian Scientific Research Fund (OTKA) under contract number T046129. Zs. Sz. is supported by OTKA Postdoctoral Grant no. PD 050015. Support by the Hungarian Research and Technological Innovation Fund, and the Croatian Ministry of Science, Educa-

tion and Sports is gratefully acknowledged. The authors acknowledge valuable comments of the Referee on the paper.

\section{References}

[1] B. Patt and F. Wilczek, hep-ph/0605188

[2] X. Calmet and J. F. Oliver, hep-ph/0606209

[3] H. Davoudiasl, R. Kitano, T. Li and H. Murayama, Phys. Lett. B609, 117 (2005)

[4] J. J. van der Bij, Phys. Lett. B636, 56 (2006)

[5] M. Shaposhnikov and I. Tkachev, Phys. Lett. B639, 414 (2006)

[6] R. Barbieri and L. J. Hall, hep-ph/0510243

[7] D. G. Cerdeno, A. Dedes, T. E. J. Underwood, hep-ph/0607157

[8] A. Hill and J. J. van der Bij, Phys. Rev. D 36, 3463 (1987)

[9] R. S. Chivukula, M. Golden, Phys. Lett. 267, 233 (1991)

[10] A. Yu. Ignatiev and R. R. Volkas, Phys. Lett. B487, 294 (2000)

[11] J.-P. Blaizot, E. Iancu and U. Reinosa, Nucl. Phys. A736, 149 (2004)

[12] R. J. Rivers, Path Integral Methods in Quantum Field Theory, Cambridge University Press 1987, Cambridge

[13] W. A. Bardeen and M. Moshe, Phys. Rev. D 28, 1372 (1983)

[14] S. R. Coleman and E. Weinberg, Phys. Rev. D 7, 1888 (1973) 